\title{
Implant-Associated Infections: A Review of the Safety of Cardiac Implants
}

\author{
Venkataramana Kandi $^{1}$, Sabitha Vadakedath ${ }^{2}$ \\ 1. Clinical Microbiology, Prathima Institute of Medical Sciences, Karimnagar, IND 2. Biochemistry, Prathima Institute \\ of Medical Sciences, Karimnagar, IND
}

Corresponding author: Venkataramana Kandi, ramana20021@gmail.com

\begin{abstract}
Cardiac implantations are among the most critical, and life-saving patient management procedures. Most cardiac implantations are performed to correct abnormalities in the conduction and the rhythm of the heart. Because the implants are intended for long-term use ranging from months to years, the failure of an implant is considered a major setback both in the patients as well as surgeons' perspectives. Implant failures can have multifactorial reasons, amongst which infectious causes need to be adequately addressed. This review attempts to evaluate the nature of implants, etiology, predisposing factors, infection control, and preventive strategies for cardiac implant-associated infections.
\end{abstract}

Categories: Cardiology, Infectious Disease

Keywords: cardiac implantations, rhythm of the heart, conduction, failure of implants, implant associated infections, etiology, predisposing factors, preventive strategies

\section{Introduction And Background}

Infection is a result of the invasion of microorganisms into the host. The infections are either endogenous or exogenous, wherein the exogenous infections occur because of the entry of microbes into the humans by various modes that include inhalation, ingestion, contact, inoculation, and from mother to the child. The endogenous infections develop in humans due to the invasion and proliferation of microbial species that are already present in the human body as commensals. Although most microbial invasions do not lead to infection, the consequence of a microbial invasion depends on several factors. The implant-associated infections (IAIs) are infections associated with the implants which are generally used to treat/manage patients. The implants are made up of metals, and other synthetic materials that may be used as a substitute, to support and balance while managing patients suffering from various conditions that include cardiac conduction abnormalities, valve dysfunctions, bone fractures, and others. The IAIs depend on the nature of the implant, the type of procedure, the experience of the surgeon, virulence determinants of the invading microbe, and the status of the host's immune system influence the IAIs. The present review delineates the nature of implants, IAIs, cardiac IAIs, etiopathogenesis, predisposing factors, control, and preventive strategies to minimize IAIs.

Review began $11 / 29 / 2020$ Review ended 12/20/2020 Published 12/25/2020

\section{(c) Copyright 2020}

Kandi et al. This is an open access article distributed under the terms of the Creative Commons Attribution License CC-BY 4.0., which permits unrestricted use, distribution, and reproduction in any medium, provided the original author and source are credited.

\section{Review \\ Implants}

Implants are devices used by health professionals during patient management. An implant may be any device that is incorporated into the human body, usually temporarily, as done in the case of catheters being inserted in the patient management for various conditions. The implants may be metal devices that are used for support by the orthopedic surgeons during a bone repair/corrective surgery. Also, the dental surgeons use various types of metal, and non-metal antibiotic-impregnated and non-impregnated implants during different types of teeth, oral and maxillofacial surgeries.

An implant could be a prosthetic device that is majorly used as a support, barrier, or a substitute for a human organ like the bone, joint, breast, hip, pelvis, mitral valve, teeth, cardiac stents, biliary stents, and others. Also, implants (mesh-like, rubber, synthetic, and plastic) are available to treat various conditions that include the collapse of pelvic organs and hernias [1-3].

Conversely, an implant may be an electronic device used for the proper functioning of muscles, brain, and heart. Cardiac implants that are frequently used to treat heart patients including cardiac implantable electronic devices (CIED), such as a pacemaker, implantable cardioverter-defibrillator (ICD), implantable cardiac loop recorder, and cardiac resynchronization therapy devices $[4,5]$.

Implants for human use may be made up of metal, plastic, rubber, silicone, graphene, or animal tissue (pig tissue) [6]. The metals which are rust-free like stainless steel, titanium, gold, silver, platinum, and tungsten, and other alloy-based metals, and liquid amalgams including the chromium-cobalt, and nickel-titanium are used to prepare the implants [7]. 


\section{Cureus}

In recent research findings, the use of polymer-zinc oxide nanoparticle composite films in the preparation of cardiac implants that function as electronic devices is recommended. This material was found to have improved insulating capabilities, and do not react or interfere either with other host biofluids/environment and electrically active human tissues like the brain and spinal cord [8].

The major concern arising from the presence of an implant in human beings is the allergic/hypersensitive reaction and inflammatory response that may be mounted by the host's immune system against the implant/device [6].

Since an implant remains for a long period ranging from a few months to several years, the occurrence of IAIs and their potential to cause severe morbidity is often a cause for serious concern [9]. There are several reports of IAIs resulting in severe morbidity and mortality $[10,11]$

\section{Etiopathogenesis}

The implants act as foreign surfaces in the human body, thereby facilitating the colonization of microbial species, that hitherto is cleared by the host's innate immune mechanisms. The reason for this is the fact that the bacteria that adhere to such implant surfaces are less susceptible to killing/elimination by phagocytosis. Also, the bacteria survive on the implant surfaces and develop biofilms that reduce the effect of antimicrobial agents and result in persistent colonization [12]. Bacteria that form biofilms were noted to demonstrate increased antimicrobial resistance and infections by such microbes are difficult to treat [13]. The microbial origins and the characteristics that facilitate IAIs are shown in Figure 1.

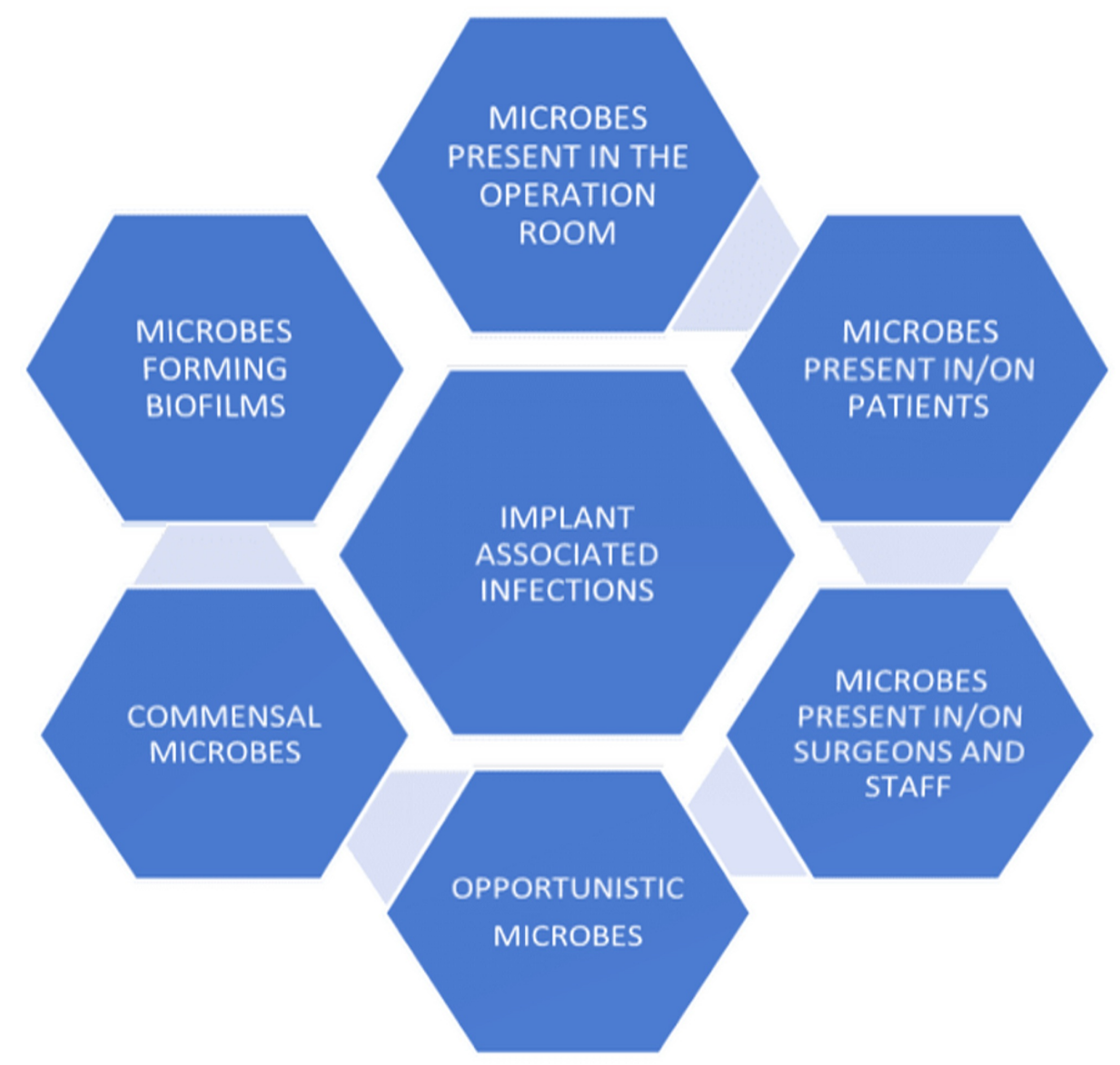

FIGURE 1: The microbial origins and characteristics that facilitate IAls

IAls: implant-associated infections

The adherence of bacteria to the implant surfaces is facilitated by the presence of fimbriae, and surface proteins like fibronectin, fibrinogen, and collagen-binding proteins, and microbial surface components recognizing adhesive matrix molecules (MSCRAMM) [14,15].

The microbes including the Staphylococcus species (sp.), Streptococcus sp., Corynebacterium sp., 
Propionibacterium acnes (Gram-positive), Escherichia coli, Pseudomonas sp., Klebsiella pneumoniae, Providentia stuartii (Gram-negative), some fungi, and Mycobacterium sp. can form biofilms and thereby pose an increased risk of IAIs $[16,17]$. Previous studies have noted that cardiac implants could pose an increased risk of infections with up to $40 \%$ of ventricular assisted device (VAD) patients developing implant-related infections $[18,19]$.

These bacteria which form biofilms on the implants elicit almost continuous inflammatory responses, thereby creating an environment that potentially causes failure of the implant [20].

An evaluation of stent associated respiratory tract infections (SARTI) among patients with stent implantation revealed that S. aureus (50\%) and Pseudomonas aeruginosa (35.7\%) were predominant pathogens followed by Candida albicans [21]. Infective endocarditis caused by Gram-negative bacteria like $E$. coli, $P$. aeruginosa, and K. pneumoniae, were frequent among cardiac implant patients with immunosuppression and chronic genitourinary colonization of bacteria [22].

Methicillin-resistant S. aureus (MRSA) (23.4\%) was the most frequent cause of cardiac permanent pacemaker associated infection followed by Methicillin sensitive S. aureus (14.9\%), Pseudomonas (10.6\%), E. coli (8.5\%), and Klebsiella (6.4\%) [23]. S. aureus and S. epidermidis were the two frequently isolated bacteria associated with cardiac IAIs among patients who were implanted with both new and re-used pacemakers and defibrillators [24].

The occurrence of microbes having the ability to grow slowly and cause persistent infections which are difficult to treat by traditional antimicrobial therapy regimens may pose an increased threat in debilitated patients that include patients with cardiac implants. Such variants of microbes are referred to as small colony variants (SCVs). Interestingly, most common IAI causing microbes like S. aureus, E. coli, and Pseudomonas sp. have been noted to form SCVs [25].

Most microbial species that are frequently associated with IAIs can produce slime, and capsules which help them to adhere to host cells, and implant surfaces thereby evade inhibition and neutralization by phagocytosis, complement-mediated lysis, and other immunological responses. Also, such microbial species resist killing by antimicrobial therapeutic agents [26].

\section{Predisposing factors}

The IAIs form an integral part of hospital-acquired/associated infections (HAIs) [27]. Among the various factors that predispose to IAIs, the nature of the implant assumes increased significance. Implants are made of polyvinyl chloride, polyethylene, latex, silicone, and stainless steel material. Implants with a rough texture and irregular surfaces favor bacterial colonization as compared to the smooth and hydrophilic implants [10]. Synthetic materials have been noted to encourage bacterial adherence and colonization as compared to biomaterials as shown in Figure 2.

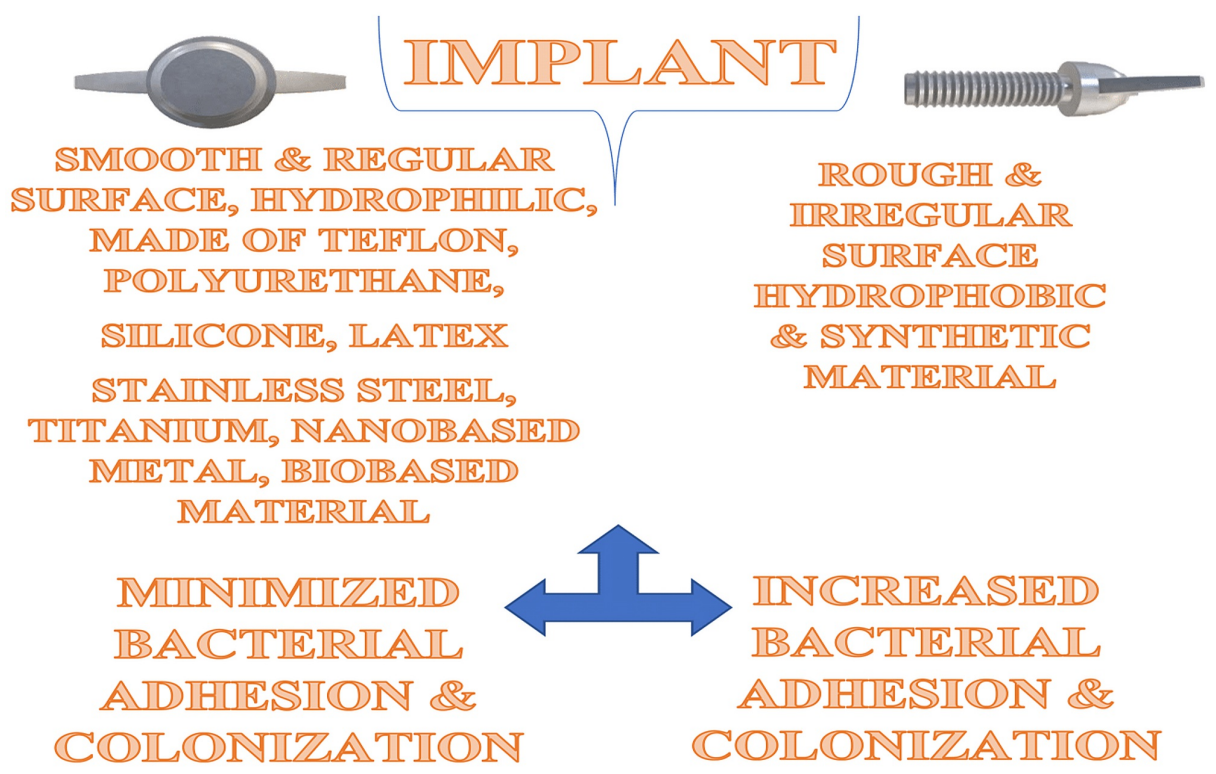

FIGURE 2: The characteristics of implants 
Factors that predispose cardiac implant patients to infections include extremes of age, presence of comorbidities like diabetes mellitus, congestive heart failure, impaired renal function, malignancy, use of immunosuppressive drugs, corticosteroids, and anticoagulants [28,29].

A recent study that evaluated the frequency, etiology, and risk factors for left ventricular assist device (LVAD)-associated infections found that more than $30 \%$ of patients developed IAIs. The most frequently isolated bacterium was S. aureus (45.4\%), followed by the Enterobacteriaceae members (24.6\%), P. aeruginosa (13.7\%), and coagulase-negative Staphylococcus (CONS) (5.2\%). About 5.2\% of patients had reportedly suffered IAI caused by Candida sp. Increased age ( $>58$ years) and the type of implant were considered as the risk factors for IAIs [30].

Predisposing factors related to the CIED related infections were systematically reviewed in a previous study. This study had noted that the IAIs could be associated with the host factors, the operative procedures (pre-, peri-, and post-operative), and the implantable device factors. The host factors that predispose to IAIs include diabetes mellitus, end-stage renal disease (ESRD), chronic obstructive pulmonary disease, corticosteroid and anticoagulant therapy, history of a previous device-associated infection, heart failure, renal insufficiency, pre-surgical fever episodes, and skin disorders. The procedure-related causes for IAIs included the inexperienced surgeon, implant replacement/revision procedures, lack of adequate antibiotic therapy prior, and during the procedures [31].

The procedural perforation of the right ventricle in patients who were undergoing pacemaker implantation that leads to pericardial effusion and cardiac tamponade was recently reported $[32,33]$. These case studies highlight the role of procedural complications and the role of iatrogens/surgeons in the development of IAIs.

In a recent study, the presence of pocket hematoma was significantly associated with wound and cardiac IAIs [34]. The IAIs may be associated with the patient factors, the procedural and personnel issues, the microbial factors, and the nature of the implant as shown in Figure 3.

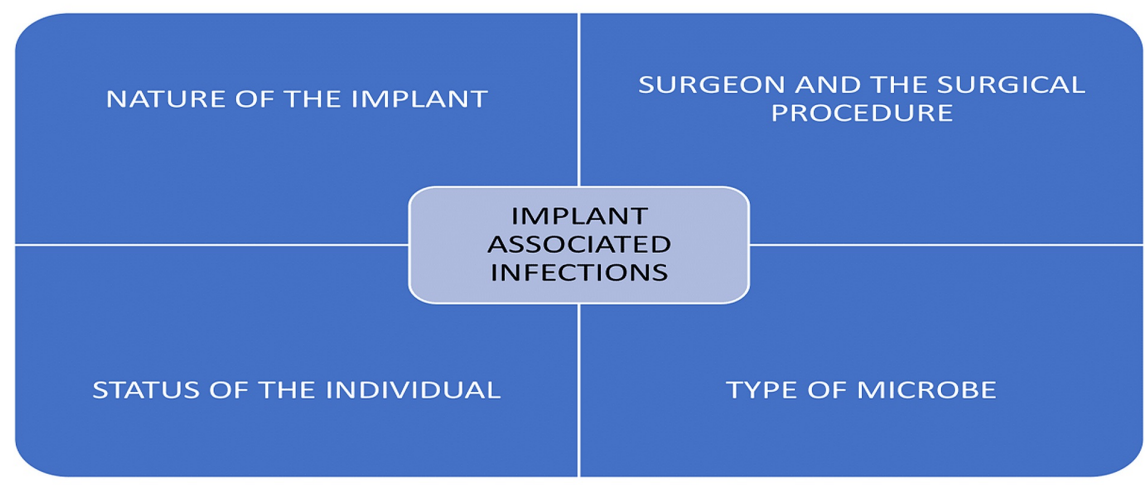

\section{FIGURE 3: The causes of IAls}

IAls: implant-associated infections

Complications arising from cardiac implants were assessed in a previous study. Majorly, the implantassociated technical failures, loosening/dislodging of the implants from the site, pocket erosion, and other adverse clinical events like cardiac tamponade, perforation, hematomas, pneumo/hemothorax, and thrombotic, and thromboembolic consequences were noted in cardiac implant patients. Also, it was noted that infection was another significant complication associated with cardiac implantations as evidenced by the occurrence of pain, peripheral nerve injury, phlebitis, and cellulitis [35].

\section{Infection control and preventive measures}

Because the implants may be colonized by the microbes with the help of biofilms, a previous study had reported the benefits of an alloy silver-coated titanium-aluminum-niobium (TiAlNb) as an implant material that could potentially minimize the colonization and thereby reduce the rates of IAIs. This study had also observed that the implants coated with antibiotics like vancomycin and daptomycin minimized the colonization of multi-drug resistant (MDR) bacteria like the MRSA [36].

Several pre-, peri-, and post-operative procedures are recommended to minimize the incidences of IAIs among cardiac implant surgical patients. Systemic antibiotic prophylaxis, a thorough screening of patients 
for remote infections, preoperative antiseptic shower, decontamination of skin at the operation/incision site, and use of protective barriers like gloves, and masks were recommended to prevent implant-related postsurgical complications [28].

The efficiency of liquid-infused surface materials when coated on the implants and other medical devices minimized the microbial adhesion and colonization. Such materials are also found efficient for drug delivery [37].

The European Heart Rhythm Association (EHRA) has recently collaborated with the European Association for Cardio-Thoracic Surgery (EACTS) and framed an international consensus document that is endorsed by the Heart Rhythm Society (HRS), the Asia Pacific Heart Rhythm Society (APHRS), the Latin American Heart Rhythm Society (LAHRS), International Society for Cardiovascular Infectious Diseases (ISCVID) and the European Society of Clinical Microbiology and Infectious Diseases (ESCMID). This document incorporates elaborate guidelines and suggestions to prevent, diagnose, and treat IAIs. It emphasizes the need for continuous monitoring of implant patients by maintaining a registry both at the national and international levels. Also, this document recommends research evidence for the use of antibiotic coated implants in minimizing infections and implant failures, duration of postoperative antibiotic therapy, appropriate timing for reimplantation surgeries, and the long term risk of death due to implant infection-related and other complications [38].

The comprehensive bundle approach that recommends the use of novel minocycline and rifampicin antimicrobial mesh (TYRX) to prevent the IAIs in patients suffering from both solid organ and hematologic malignancies with CIED was reported recently [39]. Such an approach was previously confirmed as an important strategy to prevent cardiac IAIs as evidenced by the results of the Worldwide Randomized Antibiotic Envelope Infection Prevention Trial (WRAP-IT). This trial recommends the use of TYRX, an antibiotic eluting mechanism that releases the antimicrobial agent into the surgical pocket for a minimum of seven days and thereby prevents IAIs among high-risk patients including cancer patients [40]

Miniaturized and micro implantable devices used to treat cardiac conditions were noted to substantially minimize the risk of complications and infection-related hospitalizations among patients [5].

Currently, no evidence supports the prescription of antibiotics after implant surgery. A recent study had attempted to evaluate the patients who have been prescribed both presurgical and postsurgical antibiotics after cardiac implant surgery. This study had found no significant difference of susceptibility to infections in patients who were given presurgical antibiotic course and those who received both presurgical and postsurgical antibiotic therapy. Also, it was suggested that postsurgical antibiotics must be prescribed only after considering patient factors that include age, presence of co-morbidities, and others [41].

A more than two-fold increased risk of acquiring infections is associated with the cardiac device implantation, replacement, and repair surgeries. It is important to identify patients at risk, diagnose the infection using pathogen detection in blood cultures, and finding pathogen biofilms by imaging techniques and initiate appropriate and specific antimicrobial therapy to minimize the morbidity and mortality associated with IAIs [42-44].

Strict adherence to infection control procedures during implantation surgeries could prevent the contamination of implant by skin flora and the use of antimicrobial enveloped implants minimize the chances of IAIs [45].

A recent study had suggested that although several factors including the patient, procedural, and others that contribute to IAIs, the use of anticoagulant and antiplatelet therapy could reduce the risk of cardiac IAIs [46].

\section{Conclusions}

The cause of IAIs seems to be multifactorial, wherein the patient factors, the surgeon, and procedural causes, the nature of the implant, the source, type, and the ability of the microbes may all influence the occurrence of IAIs. The IAIs could be controlled and minimized by using improved implant materials that include nano-based materials, biobased implants, leadless implants, and implants precoated with antibiotics. Also, the IAIs may be prevented by thoroughly screening the patients and operation theatre staff for microbes colonized in the mucosal surfaces and the skin, clinically evaluating the patients for prior bacteremia, fever episodes, and potential co-morbidities prior to implantation procedures, and timely initiation of preprocedural antibiotic therapy.

\section{Additional Information \\ Disclosures}

Conflicts of interest: In compliance with the ICMJE uniform disclosure form, all authors declare the following: Payment/services info: All authors have declared that no financial support was received from 
any organization for the submitted work. Financial relationships: All authors have declared that they have no financial relationships at present or within the previous three years with any organizations that might have an interest in the submitted work. Other relationships: All authors have declared that there are no other relationships or activities that could appear to have influenced the submitted work.

\section{References}

1. Chung SH, Kim WB: Various approaches and treatments for pelvic organ prolapse in women . J Menopausal Med. 2018, 24:155-162. 10.6118/jmm.2018.24.3.155

2. Hoare BS, Khan YS: Anatomy, Abdomen and Pelvis, Female Internal Genitals. [Updated July 31, 2020] . Hoare BS, Khan YS (ed): StatPearls Publishing, Treasure Island, FL; 2020.

3. Jones HG, Rees M, Aboumarzouk OM, et al.: Prosthetic mesh placement for the prevention of parastomal herniation. Cochrane Database Syst Rev. 2018, 7:CD008905. 10.1002/14651858.CD008905.pub3

4. Types of cardiac devices . (2020). Accessed: November 20, 2020: https://nyulangone.org/conditions/cardiacdevice-management-in-adults/types.

5. Rav Acha M, Soifer E, Hasin T: Cardiac implantable electronic miniaturized and micro devices . Micromachines (Basel). 2020, 11:902. 10.3390/mi11100902

6. Statement from FDA Commissioner Scott Gottlieb, M.D. and Jeff Shuren, M.D., Director of the Center for Devices and Radiological Health, on efforts to evaluate materials in medical devices to address potential safety questions. (2019). Accessed: November 20, 2020: https://www.fda.gov/news-events/pressannouncements/statement-fda-commissioner-scott-gottlieb-md-and-jeff-shuren-md-d....

7. Metals used in medical devices . (2020). Accessed: November 20, 2020: https://www.fda.gov/medicaldevices/products-and-medical-procedures/metals-used-medical-devices\#: :text=The\%20most\%20....

8. Al-Jumaili A, Kumar A, Bazaka K, Jacob MV: Electrically insulating plasma polymer/ZnO composite films . Materials (Basel). 2019, 12:3099. 10.3390/ma12193099

9. Weinstein RA, Darouiche RO: Device-associated infections: a macroproblem that starts with microadherence. Clin Infect Dis. 2001, 33:1567-1572. 10.1086/323130

10. Cardiac implantable electronic devices (CIEDs). J Arrhythm. 2019, 35:328-472. 10.1002/joa3.12273

11. Portillo ME, Corvec S, Borens O, Trampuz A: Propionibacterium acnes: an underestimated pathogen in implant-associated infections. Biomed Res Int. 2013, 2013:804391. 10.1155/2013/804391

12. Zimmerli W, Sendi P: Pathogenesis of implant-associated infection: the role of the host . Semin Immunopathol. 2011, 33:295-306. 10.1007/s00281-011-0275-7

13. Kandi V: Biofilm production correlating with multidrug resistance among clinical isolates of Acinetobacter baumannii. J Clin Diagn Res. 2015, 9:DJ02. 10.7860/JCDR/2015/13069.6084

14. Darouiche RO, Landon GC, Patti GM, et al.: Role of Staphylococcus aureus surface adhesins in orthopaedic device infections: are results model-dependent?. J Med Microbiol. 1997, 46:75-79. 10.1099/00222615-46-175

15. Mobley HLT, Chippendale GR, Tenney JH, Mayrer AR, Crisp LJ, Penner JL, Warren JW: MR/K hemagglutinin of Providencia stuartii correlates with adherence to catheters and with persistence in catheter-associated bacteriuria. J Infect Dis. 1988, 157:264-271. 10.1093/infdis/157.2.264

16. Preda VG, Săndulescu O: Communication is the key: biofilms, quorum sensing, formation and prevention . Discoveries (Craiova). 2019, 7:e100. 10.15190/d.2019.13

17. Aryana A, Keifer T, Bhaskar R, Bowers MR, O'Neill PG, Brewer Z: Acute spontaneous erosion of the right ventricle by an infected but chronically stable defibrillator lead manifesting as cardiac tamponade. HeartRhythm Case Rep. 2020, 6:875-878. 10.1016/j.hrcr.2020.09.001

18. Fleming D, Rumbaugh K: The consequences of biofilm dispersal on the host . Sci Rep. 2018, 8:10738. 10.1038/s41598-018-29121-2

19. Jamal M, Ahmad W, Andleeb S, et al.: Bacterial biofilm and associated infections. J Chin Med Assoc. 2018, 81:7-11. 10.1016/j.jcma.2017.07.012

20. Wagner C, Hänsch GM: Pathophysiologie der implantatassoziierten Infektion: vom Biofilm zur Osteolyse und septischen Lockerung [Pathophysiology of implant-associated infections: from biofilm to osteolysis and septic loosening]. [Article in German]. Orthopade. 2015, 44:967-973. 10.1007/s00132-015-3183-Z

21. Zhang JL, Zou H, Wang HW, et al.: Incidence, bacterial spectrum and predictive risk factors for stent associated respiratory tract infection. [Article in Chinese]. Zhonghua Jie He He Hu Xi Za Zhi. 2016, 39:368371. 10.3760/cma.j.issn.1001-0939.2016.05.007

22. Falcone M, Tiseo G, Durante-Mangoni E, et al.: Risk factors and outcomes of endocarditis due to non-HACEK gram-negative bacilli: data from the prospective multicenter Italian endocarditis study cohort. Antimicrob Agents Chemother. 2018, 62:e02208-17. 10.1128/AAC.02208-17

23. Shah H, Mehmood M, Salahudin M, Altaf A: Risk factor for cardiac permanent pacemaker infection . J Nepal Health Res Counc. 2019, 17:158-162. 10.33314/jnhrc.v0i0.1272

24. Khairy TF, Lupien MA, Nava S, et al.: Infections associated with resterilized pacemakers and defibrillators . N Engl J Med. 2020, 382:1823-1831. 10.1056/NEJMoa1813876

25. Venkataramana V: Small colony variants and their clinical significance . Ann Trop Med Public Health. 2016, 9:137-139. 10.4103/1755-6783.177406

26. Kandi V: Bacterial capsule, colony morphology, functions, and its relation to virulence and diagnosis . Ann Trop Med Public Health. 2015, 8:151-153. 10.4103/1755-6783.162409

27. Boev C, Kiss E: Hospital-acquired infections: current trends and prevention. Crit Care Nurs Clin North Am. 2017, 29:51-65. 10.1016/i.cnc.2016.09.012

28. Imai K: Perioperative management for the prevention of bacterial infection in cardiac implantable electronic device placement. J Arrhythm. 2016, 32:283-286. 10.1016/j.joa.2015.06.007

29. Birnie DH, Wang J, Alings M, et al.: Risk factors for infections involving cardiac implanted electronic devices. J Am Coll Cardiol. 2019, 74:2845-2854. 10.1016/j.jacc.2019.09.060

30. Tattevin P, Flécher E, Auffret V, et al.: Risk factors and prognostic impact of left ventricular assist deviceassociated infections. Am Heart J. 2019, 214:69-76. 10.1016/j.ahj.2019.04.021 
31. Polyzos KA, Konstantelias AA, Falagas ME: Risk factors for cardiac implantable electronic device infection: a systematic review and meta-analysis. Europace. 2015, 17:767-777. 10.1093/europace/euv053

32. Philip A: Iatrogenic microperforation linked to hemorrhagic pericardial effusion complicated by cardiac tamponade. Cureus. 2020, 12:e9023. 10.7759/cureus.9023

33. Khalid M, Murtaza G, Ayub MT, Ramu V, Paul T: Right ventricle perforation post pacemaker insertion complicated with cardiac tamponade. Cureus. 2018, 10:e2266. 10.7759/cureus.2266

34. Song J, Tark A, Larson EL: The relationship between pocket hematoma and risk of wound infection among patients with a cardiovascular implantable electronic device: an integrative review. Heart Lung. 2020, 49:9298. 10.1016/j.hrtlng.2019.09.009

35. Carrión-Camacho MR, Marín-León I, Molina-Doñoro JM, González-López JR: Safety of permanent pacemaker implantation: a prospective study. J Clin Med. 2019, 8:35. 10.3390/jcm8010035

36. Kuehl R, Brunetto PS, Woischnig AK, et al.: Preventing implant-associated infections by silver coating . Antimicrob Agents Chemother. 2016, 60:2467-2475. 10.1128/AAC.02934-15

37. Howell C, Grinthal A, Sunny S, Aizenberg M, Aizenberg J: Designing liquid-infused surfaces for medical applications: a review. Adv Mater. 2018, 30:e1802724. 10.1002/adma.201802724

38. Blomström-Lundqvist C, Traykov V, Erba PA, et al.: European Heart Rhythm Association (EHRA) international consensus document on how to prevent, diagnose, and treat cardiac implantable electronic device infections-endorsed by the Heart Rhythm Society (HRS), the Asia Pacific Heart Rhythm Society (APHRS), the Latin American Heart Rhythm Society (LAHRS), International Society for Cardiovascular Infectious Diseases (ISCVID) and the European Society of Clinical Microbiology and Infectious Diseases (ESCMID) in collaboration with the European Association for Cardio-Thoracic Surgery (EACTS). Europace. 2020, 22:515-549. 10.1093/europace/euz246

39. Khalil M, Karimzad K, Durand JB, Malek AE, Raad II, Viola GM: Prevention of cardiac implantable electronic device-related infection in patients with cancer: the role of a comprehensive prophylactic bundle approach that includes the antimicrobial mesh. Open Forum Infect Dis. 2020, 7:ofaa433. 10.1093/ofid/ofaa433

40. Tarakji KG, Mittal S, Kennergren C, et al.: Antibacterial envelope to prevent cardiac implantable device infection. N Engl J Med. 2019, 380:1895-1905. 10.1056/NEJMoa1901111

41. Kabulski GM, Northup A, Wiggins BS: Postoperative antibiotic prophylaxis following cardiac implantable electronic device placement. J Innov Card Rhythm Manag. 2019, 10:3777-3784. 10.19102/icrm.2019.100804

42. Döring M, Richter S, Hindricks G: The diagnosis and treatment of pacemaker-associated infection . Dtsch Arztebl Int. 2018, 115:445-452. 10.3238/arztebl.2018.0445

43. Arnold CJ, Chu VH: Cardiovascular implantable electronic device infections . Infect Dis Clin North Am. 2018, 32:811-825. 10.1016/j.idc.2018.06.004

44. Olsen T, Jørgensen OD, Nielsen JC, Thøgersen AM, Philbert BT, Johansen JB: Incidence of device-related infection in 97750 patients: clinical data from the complete Danish device-cohort (1982-2018). Eur Heart J. 2019, 40:1862-1869. 10.1093/eurheartj/ehz316

45. Palraj BR, Farid S, Sohail MR: Strategies to prevent infections associated with cardiovascular implantable electronic devices. Expert Rev Med Devices. 2017, 14:371-381. 10.1080/17434440.2017.1322506

46. Polewczyk A, Jacheć W, Polewczyk AM, Tomasik A, Janion M, Kutarski A: Infectious complications in patients with cardiac implantable electronic devices: risk factors, prevention, and prognosis. Pol Arch Intern Med. 2017, 127:597-607. 10.20452/pamw.4065 